18 Roper, B A, British fournal of Hospital Medicine, 1973, 9, 85.

19 Department of Education and Science, Speech Therapy Services. London, HMSO, 1972.

20 Hopkins, A, Health Trends, 1975, 7, 58.

${ }^{21}$ Sarno, M T, Silverman, M, and Sands, E, fournal of Speech and Hearing Research, 1970, 13, 607.

22 Wepman, J M, fournal of Speech and Hearing Disorders, 1972, 307, 203.

\section{Transfusion malaria in developing countries}

Transfusion malaria remains a problem in developing countries, and Edrissian ${ }^{1}$ has reviewed 111 cases of transfusion malaria recorded in Iran from 1963 to 1972, while Wickramasinghe $^{2}$ has recently dealt with 16 cases of accidental transfusion malaria that occurred in Sri Lanka. Cases have been reported from a large number of countries, some of the early accounts having come from China. ${ }^{3}$ There is little doubt, however, that transfusion malaria is still grossly underreported.

The incubation period of blood-induced infection is quite different from that of mosquito-transmitted malaria, since in the former there are no tissue stages in the liver. Both the interval between infection and the appearance of parasites in the blood-the prepatent period-and the presymptomatic period depend on four factors: the number of parasites introduced; the method of inoculation; the susceptibility of the recipient; and the length of blood storage before transfusion. Reviewing a series of over 100 cases of transfusion malaria in Rumania, Lupascu et $a l^{4}$ pointed out that $86 \%$ of all cases occurred when the blood was stored for less than five days; cases after 7-12 days were rare, while infections with the blood stored for 13 or more days were exceptional. In addition to infection from blood transfusion accidental transmission of malaria may also occur with plasma if some stray red blood cells happen to contain plasmodia ${ }^{5}$ or as a result.of leucocyte transfer. ${ }^{6}$ Most reported cases of transfusion malaria are due to Plasmodium malariae, followed by $P$ vivax and $P$ falciparum.

Neither systematic screening-with various serological techniques-nor premedication of suspected donors is a practical solution in developing countries. The generally accepted procedure in areas where non-immune persons may receive blood that may possibly contain scanty malaria parasites is the prophylactic administration of antimalarial drugs to the recipients. ${ }^{78}$ As there is no likelihood of true relapses occurring after blood transmission of $P$ vivax, $P$ malariae, or $P$ ovale, radical treatment with an 8 -aminoquinoline such as primaquine is not required. A single dose of $600 \mathrm{mg}$ base chloroquine to the recipient 24 hours before or on the day of transfusion seems to protect from induced malaria. It may be prudent, however, not to rely on a single dose of chloroquine but to give all non-immune recipients the standard three-day course of antimalarial treatment. ${ }^{910}$

In parts of the world where strains resistant to the 4-aminoquinolines are known to occur, such as SE Asia and S America, the preventive administration of sulfadoxine, $1.5 \mathrm{~g}$, together with pyrimethamine, $75 \mathrm{mg}$ (Fansidar), is advisable. Once again it may be prudent to give a full three-day course of curative treatment-quinine (four doses, $540 \mathrm{mg}$ each dose, given at intervals of $12 \mathrm{hrs}$ ) and then a single dose of sulfadoxine-pyrimethamine. ${ }^{11} \mathrm{~A}$ detailed account of the problem of blood transfusion and the whole range of tropical diseases (including malaria) is available in the comprehensive review by Bruce-Chwatt. ${ }^{12}$

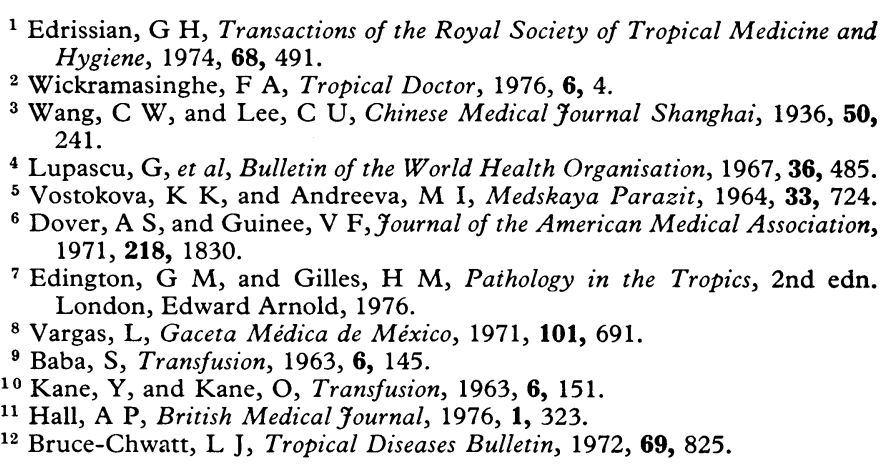

\section{Screening for presymptomatic coronary disease}

Coronary artery disease has been shown to be the cause of death in four out of every 10 men in studies with high necropsy rates. ${ }^{1}$ Yet while clinical procedures and simple laboratory tests permit recognition of traits associated with increased risk of coronary disease, ${ }^{2}$ they may not identify its presence in any one individual-let alone whether it represents a danger to him (or to others). One American man in five ${ }^{3}$ may expect to have symptoms from a coronary lesion by age 60 , but not all of those with symptoms of coronary disease will die from it, and half of those who do will never have had recognised symptoms. In these cases of unexpected sudden death there is commonly serious obstructions of two or three major coronary arteries, ${ }^{4}$ so that it follows that angina is both a poor guide to risk and an insensitive marker of underlying coronary obstructive disease. Follow-up studies after coronary angiography have shown that risk of death is closely related to the severity of coronary obstruction, ${ }^{56}$ and that the $25 \%$ or so patients with clinically diagnosed angina but normal coronary arteries ${ }^{7}$ have a normal (or better than normal) prognosis. ${ }^{6}$

Most coronary deaths are due to sudden ventricular fibrillation without infarction or occur within an hour of onset of infarction, ${ }^{8}$ but even myocardial infarction may not be a clinical event. The Framingham study showed that onequarter of the individuals who developed electrocardiographic evidence of myocardial infarction had not been recognised as having had an infarct by their doctors. ${ }^{9}$

Short of coronary angiography, then, recognising coronary disease is not always possible. An abnormal electrocardiogram may falsely suggest ischaemia, and one in four ECGs reverts to normal after actual infarction. ${ }^{9}$ Nevertheless, certain ECG abnormalities are associated with a high incidence of coronary disease. Most important of these is left ventricular hypertrophy, ${ }^{10}$ but other pointers include intraventricular conduction defects and non-specific ST and T wave changes. Changes in the ECG during or after effort lack specificity ${ }^{11}$ if an ST segment depression of $0.5 \mathrm{~mm}$ is accepted, and show inadequate sensitivity if the measure is set at $2 \mathrm{~mm}$ : at present $1 \mathrm{~mm}$ is the usual criterion. Unfortunately, the exercise ECG also suffers from lack of reproducibility: tests may vary between 
positive and negative both in individuals subsequently proved to have normal coronary arteries and (more importantly) in those with obstructive coronary artery disease or even past myocardial infarction. The problem is compounded by interand intra-observer variation in ECG reading even among experienced cardiologists. ${ }^{12}$

Until it has been shown that treatment can influence its natural history, the value of screening for presymptomatic coronary disease is arguable-except in those individuals in whom sudden death or incapacity could lead to the deaths of others. A recent report of a joint working party of the Royal College of Physicians of London and the British Cardiac Society on the care of the patient with coronary heart disease has stated that there is no currently acceptable test or combination of tests which is totally accurate in differentiating individuals with potentially dangerous coronary heart disease from those without. ${ }^{13}$ In 1965 the American College of Cardiology held its first Bethesda conference on cardiovascular problems in aviation safety and has recently reported on its eighth, ${ }^{14}$ with recommendations on criteria for acceptance, methods of continuing evaluation of flying personnel, criteria for temporary grounding and further investigation, indications for coronary arteriography, and the action to be taken on the results. Recommendations were also made for persons with proved ischaemic heart disease and, somewhat surprisingly, for postoperative patients. Commercial pilots may be recertified to fly one year after coronary artery surgery but should be re-evaluated every six months by noninvasive techniques and every two years by catheterisation.

The American report emphasised that before an aircraft is compromised not only must some incapacity affect the pilot at the controls but it must be sudden or unnoticed and take place during the critical time of landing or take off. Few serious cardiovascular incidents are known to have occurred in such circumstances: but the disaster in the Trident taking off from London airport is an example fresh in all our minds. A similar potential hazard exists among drivers of public service or heavy goods vehicles with undiagnosed coronary disease, who spend more of their time driving than does the private motorist and are therefore more likely to run into trouble while at the wheel.

What are the identifiable risk factors for such men? Morris has recently published the results of a 10-year follow-up of London busmen. ${ }^{15}$ The incidence of subsequently diagnosed disease ranged from $9 \%$ in those with normal blood pressure up to $35 \%$ in those with casual systolic blood pressure readings above $190 \mathrm{~mm} \mathrm{Hg}$. The serum cholesterol and triglyceride concentrations probably represent a similarly graded risk. ${ }^{16}$ Japan is still almost free from coronary disease despite the similar incidence to that in the West of high blood pressure, smoking, urban pollution, and stress-but there is only a $10 \%$ overlap between the lowest "normal" lipid concentrations in Britain and the highest from normal Japanese subjects. Attributes such as pure hypercholesterolaemia (Frederickson Type II or beta-hyperlipoproteinaemia) are such important predictors of premature coronary artery disease that their detection could well justify routine coronary angiography. Smoking is the third main associate of coronary disease, but any assessment of risk in an individual should also take account of factors such as lack of exercise, obesity, diabetes, and stress.

The American attitude is that coronary risk traits should be sought among flying personnel so that those at risk can be singled out for surveillance and, where possible, the traits corrected. Since 1957, when the US Air Force first demanded an electrocardiogram on entry, the requirements for professional fliers have become progressively more stringent. Pilots, engineers, navigators, and air traffic controllers are now required to submit to a full medical examination, including a baseline ECG on entry to training. Examinations are repeated with an ECG every five years to the age of 30 ; every two years to the age of 40 ; and every year to the age of 50 . Thereafter pilots have ECGs every six months. Private fliers are not required to have an ECG until the age of 40 (a serious loophole, since such men are much more likely to be flying solo). The US Air Force requires anyone with possible ECG abnormalities to have an exercise test going on to maximal treadmill exercise testing and coronary angiography in doubtful cases; but the United States Federal Aviation Administration recently withdrew a proposal for a compulsory postexercise ECG. It has been argued that to prevent one pilot from jeopardising his aeroplane 1000 pilots might be retired prematurely if abnormal postexercise ECGs became the test ${ }^{17}$; but a positive exercise test provides good grounds for coronary angiography. Even so, the results of coronary angiography carried out in 76 asymptomatic aircrew members on ECG findings showed that nearly half had normal coronary arteries; these men were recommended for return to flying duties. ${ }^{18}$ Coronary angiography can detect virtually all cases of obstructive coronary artery disease as well as of nonobstructive plaquing. Neverthless, this invasive test does carry a risk, though in experienced centres the incidence of death or serious complications should be less than $0 \cdot 1 \%$.

In Britain drivers of public service vehicles or heavy goods vehicles must submit a medical report with their first application for a licence, and medical certificates are required on the first renewal after the ages of 50, 56, 59, and 62 and annually from 65 for a public service licence, and every renewal after the age of 60 for heavy goods licence. Conditions that preclude certification include angina, a history of myocardial infarction, syncope, or arrhythmias, a blood pressure of $200 / 110 \mathrm{~mm} \mathrm{Hg}$ or over, electrocardiographic evidence of myocardial ischaemia, or radiological evidence of significant cardiac enlargement.

The importance of coronary disease as a possible cause of road accidents has been reviewed by Ysander. ${ }^{19}$ In Britain disease of any sort was considered to contribute to only 2 per 1000 of all road accidents causing personal injury. The public hazard from episodes of coronary disease in bus and lorry drivers is therefore exceedingly small in comparison with the risks from crashes due to other causes. Even so the joint working party has recommended that the requirements for successful certification should be made stricter.

The risk to the public from drivers of private cars is less. Though coronary disease is the most common cause of death from natural causes while at the wheel, Peterson and Petty reported that over half the drivers in 81 such incidents could stop their cars before the accident and that the crashes that occurred were minor and produced no serious injury to other people. ${ }^{20}$ Supported by these and similar data the Medical Commission for Accident Prevention has advised that known coronary disease should not be regarded as a bar to driving private vehicles except within two months of myocardial infarction or if driving aggravates angina. The joint working party concurred with this advice. ${ }^{13}$

\footnotetext{
1 Spierkerman, R E, et al, Circulation, 1962, 25, 57.

2 Kannel, W B, and Dawber, T R, Heart and Lung, 1972, 1, 797.

${ }^{3}$ Inter-society Commission for Heart Disease Resources, Circulation, 1970, 42, A55.

4 Vedin, J A, et al, American Heart fournal, 1973, 86, 124.

5 Oberman, A, et al, Bulletin of the New York Academy of Medicine, 1972, 48, 1109.
} 
${ }^{6}$ Bruschke, A V G, Proudfit, W L, and Sones, F M, Circulation, 1973, 47, 1147.

7 Amsterdam, E A, Wolfson, S, and Gorlin, R, American fournal of Cardiology, 1969, 23, 104

8 Gordon, T, and Kannel, W B, fournal of the American Medical Association, $1971,215,1617$.

9 Margolis, J R, et al, American fournal of Cardiology, 1973, 32, 1.

$10 \mathrm{Kannel,}$ W B, et al, Annals of Internal Medicine, 1970, 72, 813.

${ }^{11}$ Most, A S, Kemp, H G, and Gorlin, R, Annals of Internal Medicine, 1969, 71, 1043.

12 Blackburn, H, American fournal of Cardiology, 1968, 21, 871.

13 Joint Working Party, Fournal of the Royal College of Physicians of London, $1975,10,5$.

14 Task Force 1: Identification of Ischemic Heart Disease, American fournal of Cardiology, 1975, 36, 597.

15 Morris, J N, Proceedings of the Royal Society of Medicine, 1973, 66, 225.

16 Lewis, B, et al, British Medical fournal, 1974, 3, 489.

17 Garrison, G E, and Gullen, W H, Aerospace Medicine, 1972, 43, 86.

${ }_{18}$ Froelicher, V F, Jn, et al, Circulation, 1973, 48, 597.

19 Ysander, L, Acta Chirurgica Scandinavica, suppl 409.

20 Peterson, B J, and Petty, C S, Journal of Forensic Sciences, 1962, 7, 274.

\section{Injuries to the urethra}

Trauma to the urethra poses problems connected not only with the site and character of the lesion but also with the immediate and remote effects of the injury. Broadly speaking, urethral injuries mainly arise from either ill-judged or difficult instrumentation or from external violence, and their clinical importance depends on the degree of tissue damage and displacement. Relatively minor trauma may often be of little consequence (apart from sometimes leading to eventual stricture), but the immediate sequelae of more extensive laceration or rupture merit special concern, in both the short and long term. Most such cases stem from external trauma and present with the classical features of bleeding from the external meatus, inability to pass urine, and urinary extravasation or retention. Many are complicated by associated injuries-pelvic fractures, perineal lacerations, and damage to intra-abdominal tissues and viscera which themselves call for early attention. It is particularly in this context that the priorities of treatment have to be assessed, always remembering that the urethra is a vital conduit and that every effort must be made to preserve or restore its continuity at the appropriate time.

The problems of management have been admirably discussed in separate contributions by Blandy ${ }^{1}$ and Mitchell ${ }^{2}$ in a recent issue of Injury. Both authors agree on the application of basic surgical principles for the diversion of urine and drainage of extravasation whenever the continuity of the urethra was thought to be threatened. Such simple measures, indeed, may be all that can be undertaken initially in cases complicated by serious coexistent injuries or pelvic fracture and will allow some respite before deciding on the scope and quality of subsequent management. Some difference of opinion, however, was apparent on the place of urethral instrumentation or catheterisation (including urethrography) as a preliminary, especially when the injury appeared to be local. At first sight it might seem appropriate to test the continuity of the urethra and, if possible, drain the bladder in this manner; but whereas the two authors agreed on the risk of introducing infection at the traumatised area, Mitchell went further in condemning all forms of instrumentation as likely to tear any remaining strip of urethral mucosa and thus convert a partial injury into a complete rupture. Much of his experience has been concerned with pelvic injuries in which the urethra has been attenuated or dislocated above the urogenital diaphragm, and in such cases he is content to rely initially on suprapubic urinary diversion combined with appropriate orthopaedic management in the hope that some semblance of urethral continuity can be preserved.

Irrespective of this, most cases of partial rupture affecting the whole coat of the urethra eventually result in stricture, and this will clearly be more difficult to treat if healing is allowed to occur in a dislocated and unsplinted position. When there is also a pelvic fracture radiological examination may help in deciding whether serious displacement of the urethra is likely-in which case, unless demanded on account of other injuries such as suspected rupture of the bladder, retropubic exploration and reapproximation are best deferred until the immediate effects of trauma have subsided.

Ultimately many cases result in stricture, and the choice of treatment lies between repeated dilatation and urethroplasty. Whereas Mitchell claimed considerable success with endoscopic dilatation, even where residual tortuosity remains, Blandy's experience favoured the newer techniques of urethroplasty. Possibly for that reason his belief is that "the principles which underlie urethral surgery are no different from the principles which should underlie the surgery of all trauma-to remove haematoma and dead tissue, to restore anatomical continuity, and to direct urine and faeces away from the healing tissues. Displacement of the parts, organising haematoma, sepsis, and extravasation of urine will all promote scarring and make subsequent urethroplasty difficult."

Clearly much depends on the quality and extent of the urethral injury, and, while most surgeons agree on the place of conservative initial surgical management, the treatment of any resultant stricture still remains controversial.

\footnotetext{
${ }^{1}$ Blandy, J P, Injury, 1975, 7, 77.

${ }^{2}$ Mitchell, J P, Injury, 1975, 7, 84
}

\section{Structure and function in diabetic autonomic neuropathy}

The most important recent advances in our understanding of autonomic neuropathy have been the methods devised for simple clinical measurements of autonomic function. Observations of beat-to-beat variation in the heart rate ${ }^{12}$ or the rise in blood pressure during sustained hand grip ${ }^{3}$ give a measurable and reproducible assessment of autonomic defects. It is now simple to detect early abnormalities ${ }^{4}$ and to follow their progress, so that further rapid developments may be expected.

Studies of the basic mechanisms causing autonomic neuropathy have developed more slowly. The search is hampered by the inaccessibility of these nerves either for biopsy or for electrophysiological testing. The remarkably intermittent nature of the main clinical syndromes ${ }^{5}$ makes it even more difficult to relate them to anatomical and physiological abnormalities. For instance, diabetic diarrhoea is not only intermittent but also has a finite course, and it may eventually resolve. Diabetic vomiting is uncommon, but the bouts almost always terminate spontaneously. Even postural hypotension ${ }^{5}$ and gustatory sweating ${ }^{6}$ vary to a remarkable degree. Cardiac denervation, severe bladder disturbances, and neurogenic impotence are, however, permanent and irreversible. 